\title{
Status of organochlorine pesticides in the drinking water well-field located in the Delhi region of the flood plains of river Yamuna
}

\author{
P. K. Mutiyar ${ }^{1}$, A. K. Mittal ${ }^{1}$, and A. Pekdeger ${ }^{2}$ \\ ${ }^{1}$ Department of Civil Engineering, Indian Institute of Technology Delhi, 110016 New Delhi, India \\ ${ }^{2}$ Institute of Geological Sciences, Free University of Berlin, 12249 Berlin, Germany \\ Received: 18 July 2011 - Published in Drink. Water Eng. Sci. Discuss.: 12 August 2011 \\ Revised: 18 October 2011 - Accepted: 31 October 2011 - Published: 11 November 2011
}

\begin{abstract}
This study presents the occurrence of pesticides in a well-field located in Yamuna flood plain of Delhi region. Ground water sampling campaigns were carried out during pre-monsoon and post-monsoon periods covering 21 borewells and 5 Ranney wells. Major 17 organochlorine pesticides (OCP's) along with other water quality parameters were monitored during this period. Pesticide concentrations were determined using GC-ECD, while GC-MS was used for confirmatory purposes. OCP's groups like $\sum \mathrm{HCH}$, $\sum$ DDT, endosulfan and aldrin were observed in this well-field. Concentration of OCPs from Ranney well exceeded the limit $\left(1 \mu \mathrm{g} \mathrm{l}^{-1}\right)$ prescribed by the Bureau of Indian Standards (BIS) in pre-monsoon season, though OCP levels in borewells were within BIS limits. However, these levels were very close to the World Health Organisation (WHO) and European Union (EU) limit of for pesticides $\left(0.5 \mu \mathrm{gl}^{-1}\right)$ in many samples. Borewell produced better quality water compared to the water from Ranney wells. Although, the level of OCP's was slightly lower than prescribed limit of national regulatory agency but such low doses may cause long-term damage to human populations if such water is consumed for longer durations. At low doses OCP's acts as endocrine disrupting agent and cause metabolic disorders in local population.
\end{abstract}

\section{Introduction}

Every year hundreds of new molecules are produced by the industry in addition to the production of existing ones. These chemicals either in the original form or as residues enter different components of the ecosystem. Several of these synthetic organics are persistent compounds. Important groups include pesticides, polycyclic aromatic hydrocarbons (PAHs) and pharmaceutical and personal care products (PPCPs). Pesticide contamination in drinking water resources is widely recorded in many parts of the world. Majority of pesticides enter the environment through non-point sources, following normal spraying in agricultural field and further surface run-off systems. Incidences of occurrence of pesticide residues in ground water may be of prime concern because majority of rural and urban populations in the developing world depend upon ground water resources for potable purposes.

Correspondence to: P. K. Mutiyar (mutiyar_pk@yahoo.co.in)
The organochlorine groups of pesticides are nonbiodegradable and thereby these compounds can be concentrated through food chains and produce a significant magnification of the original concentration at the end of the chain. Once contaminated, (entered) these pesticides remain there for long. It is also supported by long half life of community like DDT's half life is $30 \mathrm{yr}$. Other chlorinated pesticides such as aldrin, endosulfan, $\mathrm{HCH}$, lindane, endrin and dieldrin remain stable in water for many years after their use. Due to long residence time of these substances, there is a great interest in examining their presence in the environment. Reports showing their ubiquitous presence have made the situation worse as their residues are found in water (Hassan et al., 1996), ground water (Kaushik et al., 2011; Mudiam et al., 2011), food commodities (Mukherjee and Gopal, 1996), dairy milk (John et al., 2001), bovine milk (Sharma et al., 2007), edible oil (Bajpai et al., 2007), animal feed (Mukherjee and Gopal, 1996), mother's milk (Kumar et al., 2006a), human blood (Waliszewski et al., 2001) and in skin (Dua et al., 1998). Organochlorine pesticides (OCP's) were detected even in the most remote parts of the pristine environment like 
ice core from Mt. Everest (Wang et al., 2008), Antarctic marine ecosystem (Geisz et al., 2008), Alpine glaciers (Villa et al., 2003; Bizzotto et al., 2009) and the breast milk of Inuit communities of Canada (Barrie et al., 1992; Blais et al., 2007) where these pesticides have never been used, but reached due to global circulation pattern and cold deposition on mountains. In India, OCP's have been reported in drinking water sources at: Bhopal (Dikshith et al., 1990), Hyderabad (Shukla et al., 2006), Jaipur (Bakore et al., 2004), Kanpur (Sankararamakrishnan et al., 2005) and Ahemedabad (Jani et al., 1991). The total pesticides consumption in Delhi area is continuously decreasing due to conversion of agricultural land to residential and industrial land. The total pesticides consumption in Delhi area was 100 MT 1990 which reduced to $53 \mathrm{MT}$ in 2005 (www.ncipm.org.in). Mukherjee and Gopal (2002) reported higher level $\left(\mu \mathrm{g} \mathrm{ml}^{-1}\right)$ of OCP's contamination in ground water samples taken from different zones of Delhi. But now we expected to have lower levels of pesticide residues in the ground water due to decreased pesticide consumption. It is evident from the literature that no comprehensive report is available on pesticide residues in Yamuna flood areas of Delhi region which is the main watershed zone for harvesting water for urban supply of Delhi NCT. The present study is focused on the special Palla-Burari well-field. The study area is strategically important because this area is also used by the local water supply organization as a potable water source. It has around 80 borewells and 5 Ranney wells to extract the good quality water from the Yamuna river flood plains to meet the growing water demand of Delhi. About $15 \%$ of the public water supply of Delhi is contributed by this well-field, making the area hotspot as this area contributes a significant fraction of drinking water to 16.8 million people of Delhi. Since, this well-field has both the borewells and Ranney wells, so it is also contemplated to study effect of type of well on the water quality.

\section{Materials and methods}

\subsection{Description of sampling site}

This site is located along the upstream of urbanised parts of Delhi comprising, North West and North district of Delhi, on the flood plain of the western bank of the river Yamuna. The total sampling area of well-field is around $40 \mathrm{~km}^{2}$, on the western bank of river Yamuna, starting from outskirts of Delhi (un-urbanised area) to the border of neighbouring state of Haryana. The flood plains have an embankment on both sides at a distance of 500-2000 m from the actual course of river Yamuna to protect the populated rural area from flood events. The flood plain is of younger alluvium and the sediments have been deposited upon a series of several hundreds of meters of older alluvium, which predominately composed of fine sand, silt and clay (Shekhar and Prasad, 2009). The well-field has relatively plain terrain within the embankment and the ground water level is about

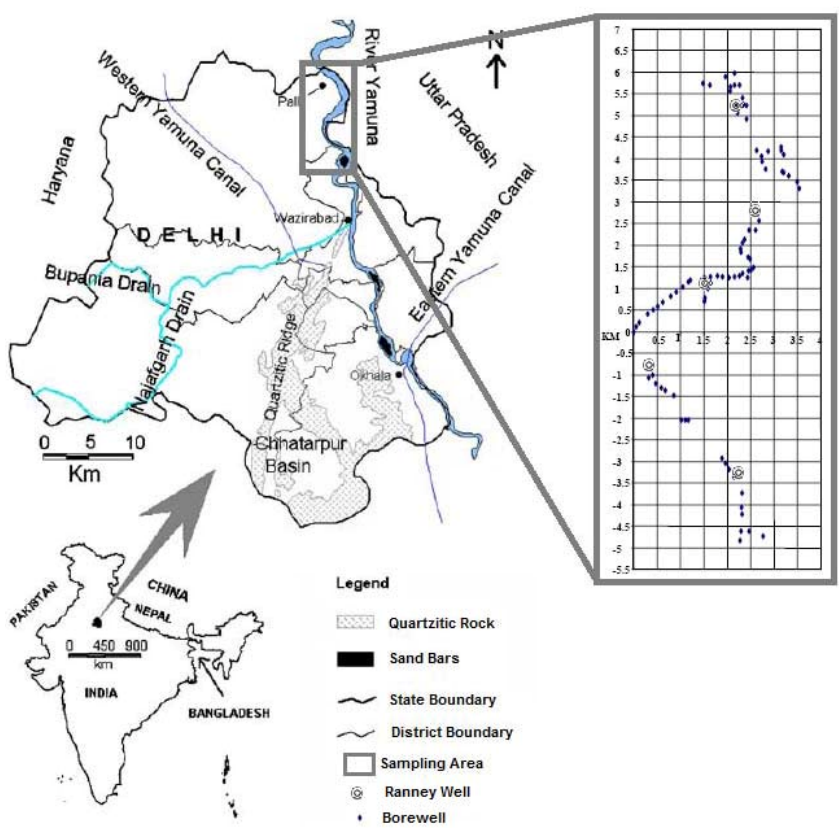

Figure 1. Map showing location of the study area (NCT Delhi) with the grids selected for the OCP analysis (map: modified from Shekhar and Prasad, 2009).

4-6 m b.g.l. (below ground level). The well-field contains two types of wells, Ranney wells and borewells. Ranney wells comprise a central concrete caisson excavated to a target depth (shallow) at which well screens project laterally outward in a radial pattern and are designed to induce infiltration. The site is on the bank of river Yamuna, thus can support Ranney wells by inducing infiltration through riverbank filtration from river Yamuna. The well-field also contains borewells which pumps the water from relatively deeper aquifer. Yield of borewells ranges from $100-280 \mathrm{~m}^{3} \mathrm{~h}^{-1}$ in the area (Kumar et al., 2006b). The distance of wells sampled, from the feeding water source Yamuna river varies from $50-1000 \mathrm{~m}$. The site is strategically important to compare the water quality from shallower and deeper aquifers, as Ranney wells water sample represent ground water quality in shallow aquifer while borewell water sample represent the deep aquifer water quality. Thus water samples were collected from borewells and Ranney wells, which are located between latitude $28^{\circ} 46^{\prime} 56.69^{\prime \prime} \mathrm{N}$, longitude $77^{\circ} 11^{\prime} 58.62^{\prime \prime} \mathrm{E}$ and latitude $28^{\circ} 50^{\prime} 34.94^{\prime \prime} \mathrm{N}$, longitude $77^{\circ} 12^{\prime} 34.91^{\prime \prime}$ E. Sampling sites are shown in Fig. 1.

\subsection{Water sampling}

Sampling area was divided in a grid of $500 \times 500 \mathrm{~m}$, to reduce the total number of samples for OCP's analyses and to make a representative sampling as the sampling points were located in close proximity. Samples were taken from all the functional borewells and Ranney wells. One sample from 
each grid was chosen for OCP analysis in order to obtain complete representation of the well-field. Average number of borewells in a grid of $2.5 \mathrm{~km}^{2}$ was around 5. Thus 21 borewells and all 5 Ranney wells were selected for OCP analysis. The average depth of the Ranney wells $(N=5)$ was $25.56 \mathrm{~m}$ while the average depth of the borewells $(N=$ 80) was $44.07 \mathrm{~m}$. Samples were collected in high density polyethylene (HDPE) amber coloured bottles with Teflon lined caps. Prior to sampling, pumps were run for at-least 10 min to clear the casing of standing water and to bring in fresh water from the aquifer. The sampling bottles were rinsed with well water and were carefully filled to overflowing, without passing air bubbles through the samples or trapping air bubbles in sealed bottles. Field blanks were prepared with distilled water and were analysed only when pesticide residues were detected in primary samples. Two samples were taken from each site during each sampling campaign. One sample was used to determine physico-chemical parameters while the second sample was used for OCP analysis. The sample collection bottles were transported in cool-box with ice packs and subsequently stored in a refrigerator at $4{ }^{\circ} \mathrm{C}$ until further analysis.

\subsection{Reagents and standards}

Analytical grade (AR) chemicals (Merck, Germany) were used throughout the study without any further purification. To prepare reagents and calibration standards, double glass distilled water was used. The glass-wares were washed with dilute nitric acid $(1.15 \mathrm{~N})$ followed by several portions of distilled water. EPA 502 Pesticide Standard Mix (49690-U) was procured from Sigma-Aldrich USA. The working standards of pesticides were prepared by diluting EPA pesticide mixture standard in $n$-hexane and were stored at $-20^{\circ} \mathrm{C}$.

\subsection{Physico-chemical parameters}

Samples were analysed for different physico-chemical parameters: $\mathrm{pH}$, electrical conductivity (EC), alkalinity, hardness, major anions $\left(\mathrm{Cl}^{-}, \mathrm{F}^{-}, \mathrm{NO}_{3}^{-}, \mathrm{SO}_{4}^{-2}\right)$ and major cations $\left(\mathrm{Na}^{+}, \mathrm{K}^{+}, \mathrm{Ca}^{+2}, \mathrm{Mg}^{+2}\right)$ as per APHA (1998). EC and $\mathrm{pH}$ were measured using a portable $\mathrm{EC}$ and $\mathrm{pH}$ meter, respectively.

\subsubsection{Extraction of pesticides residues}

Method prescribed by APHA (1998) with some modifications was used for the extraction of OCP residues from the water samples. A liquid liquid extraction (LLE) method, using $n$-hexane as solvent, was used for extraction of pesticide residues. The water samples were prefiltered using $0.45 \mu \mathrm{m}$ glass-fiber filter to remove any suspended impurity and were extracted without any $\mathrm{pH}$ adjustment. For extraction, one litre water sample was taken into a separating flask. It was mixed with $30 \mathrm{~g}$ of $\mathrm{NaCl}$ and $50 \mathrm{ml}$ of $n$-hexane. Sample was shacked properly and hexane layer was separated. This process was repeated thrice with same water sample and hexane portions were pooled and transferred to a Buchi Condenser flask through a column of anhydrous sodium sulfate and florosil mixture to remove any remaining water molecules. The extract was condensed to around $2 \mathrm{ml}$ on Rotavac and further reduced to $1 \mathrm{ml}$ under gentle $\mathrm{N}_{2}$ stream. The concentrated extract was transferred to air-tight GC vials and stored at $-20^{\circ} \mathrm{C}$ until analysis.

\subsubsection{Qualitative and quantitative analysis}

Analysis of pesticide residue was carried out on a Thermo ${ }^{\circledR}$ Trace GC, microprocessor controlled gas chromatograph, equipped with electron capture detector (ECD), having Nickel (63) foil as the electron source and auto sampler. The column specifications and operating conditions are given in Table 1. Confirmation of the identity of the OCP was done on a Mass Spectrometer (MS), coupled with Trace GC Ultra. The column type, column condition, temperature programming and injector temperature were kept the same for GC-ECD and GC-MS analysis. MS transfer line temperature and ionization source temperature were kept $280^{\circ} \mathrm{C}$ and $200^{\circ} \mathrm{C}$, respectively (Table 1 ). The samples were injected by a Thermo AS 3000 autosampler by setting injection volume of $1 \mu \mathrm{l}$ in splitless mode for each sample. Analysis was done in full scan mode, range of 50-650 units with 70-eV electron impact (EI) mode and specific ions were monitored for confirmation. The instrument was operated by Xcaliur software from Thermo Finnigan. To ensure the quality of extraction and detection procedure, 5 different concentrations of each OCP standards were mixed with distilled water, extracted by the same method, and recovery was measured. Table 2 presents retention time (RT), recovery efficiency (RE), method detection limit (MDL) and ions monitored for confirmation of different pesticides for this method. Important physico-chemical properties of investigated OCPs are given in Table 3.

\section{Result and discussion}

Physico-chemical quality of the ground water of the area is presented in Table 4. Water quality of groundwater at selected locations for major parameters: $\mathrm{pH}$, electrical conductivity (EC), alkalinity, hardness, major anions $\left(\mathrm{Cl}^{-}, \mathrm{F}^{-}\right.$, $\left.\mathrm{NO}_{3}^{-}, \mathrm{SO}_{4}^{-2}\right)$ and major cations $\left(\mathrm{Na}^{+}, \mathrm{K}^{+}, \mathrm{Ca}^{+2}, \mathrm{Mg}^{+2}\right)$ was within BIS limits (IS:10500), except for EC and hardness for Ranney wells (1934.2 $\mu \mathrm{S} \mathrm{cm}^{-1}$ and $653.6 \mathrm{mg} \mathrm{l}^{-1}$, respectively) in pre-monsoon season which is slightly higher than prescribed limits. However, the EC and hardness decreased during the post-monsoon season. This could be attributed to the recharge of aquifer by good quality rain water and high flood level in river Yamuna during monsoon season. Lorenzen et al. (2010) reported a steep gradient from surface water 
Table 1. Operating conditions used for the operation of GC-ECD and GC-MS.

\begin{tabular}{|c|c|c|}
\hline & GC-ECD & GC-MS \\
\hline Column used & $\begin{array}{l}\text { DB-5, fused silica capillary } \\
\text { column }(30 \mathrm{~m} \times 0.25 \mathrm{~mm} \text { ID, } \\
\text { film thickness } 0.25 \mu \mathrm{m})\end{array}$ & $\begin{array}{l}\text { DB-5, fused silica capillary } \\
\text { column }(30 \mathrm{~m} \times 0.25 \mathrm{~mm} \text { ID, } \\
\text { film thickness } 0.25 \mu \mathrm{m})\end{array}$ \\
\hline Injector temperature & $250^{\circ} \mathrm{C}$ & $250^{\circ} \mathrm{C}$ \\
\hline Oven programming & $\begin{array}{l}90 \text { to } 150^{\circ} \mathrm{C} \text { at } 15^{\circ} \mathrm{C} \mathrm{min}^{-1} \text {, } \\
150 \text { to } 220^{\circ} \mathrm{C} \text { at } 3^{\circ} \mathrm{C} \mathrm{min} \text { min }^{-1} \\
\text { and } 220 \text { to } 270^{\circ} \mathrm{C} \text { at } \\
5^{\circ} \mathrm{C} \min ^{-1}\end{array}$ & $\begin{array}{l}90 \text { to } 150^{\circ} \mathrm{C} \text { at } 15^{\circ} \mathrm{C} \mathrm{min}^{-1} \text {, } \\
150 \text { to } 220^{\circ} \mathrm{C} \text { at } 3^{\circ} \mathrm{C} \mathrm{min}{ }^{-1} \\
\text { and } 220 \text { to } 270^{\circ} \mathrm{C} \text { at } \\
5^{\circ} \mathrm{C} \min ^{-1}\end{array}$ \\
\hline Detector temperature & $280^{\circ} \mathrm{C}$ & - \\
\hline Carrier gas & Helium at $1.5 \mathrm{ml} \mathrm{min}^{-1}$ & Helium at $1.5 \mathrm{ml} \mathrm{min}^{-1}$ \\
\hline Makeup gas & Nitrogen at $40 \mathrm{ml} \mathrm{min}^{-1}$ & \\
\hline MS transfer line temperature & - & $280^{\circ} \mathrm{C}$ \\
\hline Ionisation source temperature & - & $200^{\circ} \mathrm{C}$ \\
\hline
\end{tabular}

Table 2. Standardisation of OCP's compounds using GC-ECD and GC-MS.

\begin{tabular}{|c|c|c|c|c|c|}
\hline Compound & RT (min) & Recovery (\%) & $R^{2}$ & $\operatorname{MDL}^{\mathrm{a}}\left(\mathrm{ng} 1^{-1}\right)$ & Selected Ions $(\mathrm{m} / \mathrm{z})$ \\
\hline$\alpha-\mathrm{HCH}(\mathrm{H} 1)$ & 12.28 & 71.28 & 0.9994 & 0.01 & \\
\hline$\beta-\mathrm{HCH}(\mathrm{H} 2)$ & 13.52 & 79.42 & 0.9984 & 0.01 & \\
\hline$\gamma-\mathrm{HCH}(\mathrm{H} 3)$ & 13.74 & 70.99 & 0.999 & 0.01 & \\
\hline$\delta$-HCH (H4) & 14.97 & 78.56 & 0.9985 & 0.01 & $\mathbf{1 8 1}, 183,109,288^{*}$ \\
\hline Heptachlor (He1) & 17.22 & 87.53 & 0.9956 & 0.01 & $\mathbf{1 0 0}, 272,274,370^{*}$ \\
\hline Aldrin (A1) & 18.99 & 146.78 & 0.9988 & 0.01 & $\mathbf{6 6}, 263,79,362^{*}$ \\
\hline Hepta-Epoxide (He2) & 21.21 & 59.55 & 0.9987 & 0.01 & $\mathbf{8 1}, 353,355,386^{*}$ \\
\hline$\alpha$-Endo (E1) & 23.15 & 107.97 & 0.9993 & 0.01 & $\mathbf{1 9 5}, 197,241,404^{*}$ \\
\hline Dieldrin (A2) & 24.6 & 123.87 & 0.9993 & 0.01 & $\mathbf{7 9}, 81,263,378^{*}$ \\
\hline $4,4^{\prime}-\mathrm{DDE}(\mathrm{D} 1)$ & 24.8 & 86.16 & 0.999 & 0.01 & $\mathbf{2 4 6}, 318,316^{*}$ \\
\hline Endrin (A3) & 25.79 & 87.70 & 0.9983 & 0.01 & $\mathbf{8 1}, 79,263,378^{*}$ \\
\hline$\beta$-Endo (E2) & 26.4 & 90.85 & 0.9978 & 0.01 & 195, 197, 241, 404* \\
\hline $4,4^{\prime}-\mathrm{DDD}(\mathrm{D} 2)$ & 27.18 & 87.47 & 0.9972 & 0.01 & 235, $237,165,318^{*}$ \\
\hline Endrin-aldehyde (A4) & 27.47 & 133.12 & 0.9951 & 0.01 & $67,345,250,378^{*}$ \\
\hline Endo-Sulfate (E3) & 28.81 & 85.80 & 0.9948 & 0.01 & $272, \mathbf{3 8 7}, 420^{*}$ \\
\hline $4,4^{\prime}$-DDT (D3) & 29.24 & 99.22 & 0.9895 & 0.01 & $\mathbf{2 3 5}, 237,352^{*}$ \\
\hline Methoxychlor (M1) & 32.28 & 95.48 & 0.9868 & 0.01 & $\mathbf{2 2 7}, 228,344^{*}$ \\
\hline
\end{tabular}

MDL $=$ methods detection limit

The ions were used for confirmation purpose only; the bold ions represent the base peak ion while the ion with a asterisk shows the molecular peak ion.

to groundwater and between shallow and deeper groundwater in this area, which is likely to be a consequence of heavy pumping in the well-field leading to high recharge rates from the river and a strong vertical flow component. Water quality improved in the post-monsoon season for all the parameters (Table 4), except for $\mathrm{NO}_{3}$, which has slightly increased in the post-monsoon season. It may be due to recharge of water from surface run-off from agricultural field. The borewell water quality was much superior to Ranney well water and was less prone to seasonal change (Table 4).
Both, borewell (TW-1 to TW-21, grid wise) and Ranney well (RW-1 to RW-5) samples were analyzed for the presence of OCP's (Fig. 1, grid). One sample from each grid $(500 \times 500 \mathrm{~m})$ of the well-field was taken. One sample may represent the whole grid since OCP's contamination may not vary within the grid. In these grids, OCP's are contributed by the anthropogenic activities only. Data revealed the trace levels of OCP's in the ground water. Tables 5 and 6 show levels of OCPs detected. In pre-monsoon, only 2 samples (TW-6, TW-9) out of 21 samples did not contain any of 17 
Table 3. Physico-chemical properties of investigated organochlorine pesticides.

\begin{tabular}{|c|c|c|c|c|c|c|c|c|c|}
\hline OCP & CAS No & $\begin{array}{l}\text { Molecular } \\
\text { weight }\end{array}$ & $\begin{array}{l}\text { Molecular } \\
\text { formula }\end{array}$ & $\begin{array}{l}\text { Density } \\
\left(\mathrm{g} \mathrm{cm}^{-3}\right)\end{array}$ & $\begin{array}{l}\text { Melting } \\
\text { Point }\left({ }^{\circ} \mathrm{C}\right)\end{array}$ & $\begin{array}{l}\text { Boiling } \\
\text { Point }\left({ }^{\circ} \mathrm{C}\right)\end{array}$ & $\begin{array}{l}\text { Water Solubility } \\
\left(\mathrm{mg} \mathrm{l}^{-1}\right)\end{array}$ & $\log K_{\mathrm{OC}}$ & $\log K_{\text {OW }}$ \\
\hline$\alpha-\mathrm{HCH}$ & $319-84-6$ & 290.8 & $\mathrm{C}_{6} \mathrm{H}_{6} \mathrm{Cl}_{6}$ & 1.87 & 159 & 288 & $0.02-2$ & $3.3-3.6$ & $3.8-5.8$ \\
\hline$\beta$-HCH & $319-85-7$ & 290.8 & $\mathrm{C}_{6} \mathrm{H}_{6} \mathrm{Cl}_{6}$ & 1.89 & 314 & 60 & $0.05-0.2$ & 3.6 & 3.78 \\
\hline$\gamma-\mathrm{HCH}$ & $58-89-9$ & 290.8 & $\mathrm{C}_{6} \mathrm{H}_{6} \mathrm{Cl}_{6}$ & 1.85 & 112.5 & 323.4 & $0.7-5.59$ & 3.57 & $3.6-3.7$ \\
\hline$\delta$ - $\mathrm{HCH}$ & $319-86-8$ & 290.8 & $\mathrm{C}_{6} \mathrm{H}_{6} \mathrm{Cl}_{6}$ & NA & 141.5 & 60 & $0.2-259$ & 3.8 & 4.14 \\
\hline Heptachlor & $76-44-8$ & 373.3 & $\mathrm{C}_{10} \mathrm{H}_{5} \mathrm{Cl}_{7}$ & 1.58 & $135-145$ & $95-96$ & $0.002-0.18$ & 4.4 & $5.2-6.1$ \\
\hline Aldrin & $309-00-2$ & 364.9 & $\mathrm{C}_{12} \mathrm{H}_{8} \mathrm{Cl}_{6}$ & 1.7 & 105 & 385 & $0.002-0.078$ & 4.68 & $5.7-7.4$ \\
\hline Hepta-Epoxide & $1024-57-3$ & 389.3 & $\mathrm{C}_{10} \mathrm{H}_{5} \mathrm{Cl}_{7} \mathrm{O}$ & 1.58 & 161 & 425 & $0.02-0.275$ & 4.48 & $4.6-5.4$ \\
\hline$\alpha$-Endo & $959-98-8$ & 406.9 & $\mathrm{C}_{9} \mathrm{H}_{6} \mathrm{Cl}_{6} \mathrm{O}_{3} \mathrm{~S}$ & 1.94 & 108 & NA & $0.05-1.73$ & 4.2 & $3.8-4.9$ \\
\hline Dieldrin & $60-57-1$ & 380.9 & $\mathrm{C}_{12} \mathrm{H}_{8} \mathrm{Cl}_{6} \mathrm{O}$ & 1.75 & 176 & 385 & $0.002-0.19$ & 5.28 & $4.5-6.2$ \\
\hline DDE & $72-55-9$ & 318 & $\mathrm{C}_{14} \mathrm{H}_{8} \mathrm{Cl}_{4}$ & 1.51 & 89 & 316 & 0.065 & 5.04 & $5.9-6.9$ \\
\hline Endrin & $72-20-8$ & 380.9 & $\mathrm{C}_{12} \mathrm{H}_{8} \mathrm{Cl}_{6} \mathrm{O}$ & 1.7 & 240 & 416 & $0.026-0.25$ & 3.97 & $4.7-5.2$ \\
\hline$\beta$-Endo & $33213-65-9$ & 406.9 & $\mathrm{C}_{9} \mathrm{H}_{6} \mathrm{Cl}_{6} \mathrm{O}_{3} \mathrm{~S}$ & 1.7 & 106 & NA & $0.2-1.6$ & 4.2 & $3.6-4.8$ \\
\hline DDD & $72-54-8$ & 321 & $\mathrm{C}_{14} \mathrm{H}_{10} \mathrm{Cl}_{4}$ & 1.385 & 59.8 & 376 & $0.016-0.09$ & 4.9 & $4.7-6.3$ \\
\hline Endrin Aldehyde & $7421-93-4$ & 382.9 & $\mathrm{C}_{12} \mathrm{H}_{8} \mathrm{Cl}_{6} \mathrm{O}$ & NA & 163 & 340 & $0.016-0.26$ & 6.33 & 6.44 \\
\hline Endo-Sulfate & $1031-07-8$ & 422.9 & $\mathrm{C}_{9} \mathrm{H}_{6} \mathrm{Cl}_{6} \mathrm{O}_{4} \mathrm{~S}$ & NA & 181 & 481 & $0.001-0.1$ & 5.9 & 6.1 \\
\hline DDT & $50-29-3$ & 354.4 & $\mathrm{C}_{14} \mathrm{H}_{9} \mathrm{Cl}_{5}$ & 1.6 & 109 & 260 & 0.003 & 5.2 & $5.4-6.9$ \\
\hline Methoxychlor & $72-43-5$ & 345.6 & $\mathrm{C}_{16} \mathrm{H}_{15} \mathrm{Cl}_{3} \mathrm{O}_{2}$ & 1.39 & $86-96$ & 436 & $0.001-0.045$ & 4.89 & 5.66 \\
\hline
\end{tabular}

NA $=$ Not available

Table 4. Water quality of ground water of the Palla well field.

\begin{tabular}{|c|c|c|c|c|c|c|c|c|c|c|c|c|c|}
\hline \multirow[t]{3}{*}{ Parameters } & \multicolumn{6}{|c|}{ Pre-monsoon Season } & \multicolumn{6}{|c|}{ Post-monsoon season } & \multirow{3}{*}{$\begin{array}{r}\text { IS } \\
\text { Standards }\end{array}$} \\
\hline & \multicolumn{3}{|c|}{ Borewell } & \multicolumn{3}{|c|}{ Ranney Well } & \multicolumn{3}{|c|}{ Borewell } & \multicolumn{3}{|c|}{ Ranney Well } & \\
\hline & Ranges & Avg. & SD & Ranges & Avg. & SD & Ranges & Avg. & $\mathrm{SD}$ & Ranges & Avg. & SD & \\
\hline $\mathrm{pH}$ & $6.7-7.5$ & 7.1 & 0.2 & $6.7-7.3$ & 6.9 & 0.2 & $7.2-7.9$ & 7.5 & 0.2 & $7.2-7.4$ & 7.3 & 0.1 & $6.5-8.5$ \\
\hline $\mathrm{EC}\left(\mu \mathrm{S} \mathrm{cm}^{-1}\right)$ & $87-1770$ & 725.3 & 309.4 & $825-4030$ & 1934.2 & 1451.7 & $440-1570$ & 728.6 & 248.5 & $847-2330$ & 1210.6 & 470 & 1500 \\
\hline Sulfate $\left(\mathrm{mg} \mathrm{l}^{-1}\right)$ & $43.6-187.7$ & 83.8 & 42.4 & $59.7-255.8$ & 160.5 & 80.8 & $7-199.8$ & 54. & 43.9 & $39.2-255.7$ & 93.9 & 94.7 & $200-400$ \\
\hline $\mathrm{Cl}^{-}\left(\mathrm{mg} \mathrm{l}^{-1}\right)$ & $38-232.3$ & 86.4 & 38.3 & $72.8-1063.5$ & 369.3 & 425.7 & $32-503$ & 129.2 & 113.5 & $64-950$ & 292.6 & 414.3 & $250-1000$ \\
\hline Nitrate $\left(\mathrm{mg} \mathrm{l}^{-1}\right)$ & $0.4-0.9$ & 0.6 & 0.1 & $0.4-7.9$ & 2.2 & 3.2 & $2.1-30$ & 6.9 & 5.4 & $3.6-7.9$ & 3.5 & 1.6 & $<45$ \\
\hline $\mathrm{F}^{-}\left(\mathrm{mg} \mathrm{l}^{-1}\right)$ & $0.3-1.8$ & 0.8 & 0.4 & $0.2-0.6$ & 0.4 & 0.2 & $0.1-1.7$ & 0.6 & 0.3 & $0.2-0.4$ & 0.3 & 0.1 & $1-1.5$ \\
\hline $\mathrm{Na}^{+}\left(\mathrm{mg} \mathrm{l}^{-1}\right)$ & $28-122$ & 54.1 & 20.3 & $44-491.5$ & 223.1 & 227.0 & $7-199.8$ & 70.7 & 43.9 & $35.4-491.5$ & 119.6 & 192.2 & - \\
\hline $\mathrm{K}^{+}\left(\mathrm{mg} \mathrm{l}^{-1}\right)$ & $2-31$ & 3.7 & 4.8 & 3-8.1 & 5.0 & 2.0 & $1.5-8.4$ & 7.3 & 1.2 & $4.7-8.1$ & 9.6 & 1.3 & - \\
\hline Hardness $\left(\mathrm{mg} \mathrm{l}^{-1}\right)$ & $184-792$ & 353.7 & 178.8 & $252-960$ & 653.6 & 308.5 & $180-584$ & 260.1 & 73 & $337-484$ & 400 & 53.3 & $300-600$ \\
\hline Alkalinity $\left(\mathrm{mg} \mathrm{l}^{-1}\right)$ & $176-356$ & 241.8 & 51.0 & $244-320$ & 290.4 & 34.6 & $180-388$ & 259.2 & 56.8 & $284-336$ & 300 & 21.8 & $200-600$ \\
\hline
\end{tabular}

targeted OCP's (Fig. 2). Rest of the samples contained 2 or more pesticides. Residues of all 17 targeted OCP's were found in 3 samples only. The most frequently occurring pesticides in this sampling campaign were aldrin, followed by $\sum \mathrm{HCH}$, endosulfan and degradation product of DDT. The total numbers of pesticide occurrences were more in Ranney well, with an average of 11 OCP's per sample compared to the 7 OCP's in the borewell. None of the Ranney well sample was free from all the pesticides (Fig. 3). Concentrations of $\sum \mathrm{HCH}$ in the borewell water varied from not detected (ND) to $0.269 \mu \mathrm{gl} l^{-1}$, with maximum of $\beta$-HCH being $0.201 \mu \mathrm{g}^{-1}$ as individual $\mathrm{HCH}$. The aldrin residues were detected in the range of ND to $0.047 \mu \mathrm{g} 1^{-1}$, while dieldrin, endrin and endrin aldehyde were found in same range ND to $0.094 \mu \mathrm{g}^{-1}$ but the frequency of occurrences was $27 \%$ as compare to aldrin showing $76 \%$ occurrences. During the sampling, it was noticed that the area has problem of mites and root worms. High occurrence of aldrin could be due to the use of pesticide to control the mites in the past. In case of Ranney wells, the most frequently occurring contaminants were $\sum \mathrm{HCH}$, aldrin, DDE (frequency $=100 \%$ ), followed by $\beta$-endosulfan and methoxychlore with $80 \%$ occurrence frequency. The maximum residue of single pesticide detected were similar to borewell water i.e. $\beta$ - $\mathrm{HCH}$, $\left(0.206 \mu \mathrm{g}^{-1}\right)$. The maximum concentration of endosulfan was $0.095 \mu \mathrm{g}^{-1}$ for $\beta$-endosulfan in all the samples. During post-monsoon sampling campaign, more grids reported no pesticides (Fig. 4) and lower occurrences frequency where only 1 sample showed the presences of all the targeted OCP (Table 7) while in pre-monsoon periods 3 samples showed presence of all OCP's (Table 5). All the banned pesticides, viz $\sum \mathrm{HCH}$, aldrin, heptachlor, DDT, DDD and DDE 
Table 5. Organochlorine residues $\left(\mu \mathrm{g} \mathrm{l}^{-1}\right)$ in ground water (bore well) of Palla area during pre-monsoon seasons.

\begin{tabular}{|c|c|c|c|c|c|c|c|c|c|c|c|c|c|c|c|c|c|}
\hline & $\begin{array}{l}\alpha- \\
\mathrm{HCH}\end{array}$ & $\begin{array}{l}\beta- \\
\mathrm{HCH}\end{array}$ & $\begin{array}{l}\gamma- \\
\mathrm{HCH}\end{array}$ & $\begin{array}{l}\delta- \\
\mathrm{HCH}\end{array}$ & $\begin{array}{l}\text { Hepta } \\
\text { chlor }\end{array}$ & Aldrin & $\begin{array}{l}\text { Hepta- } \\
\text { Epoxide }\end{array}$ & $\begin{array}{l}\alpha- \\
\text { Endo }\end{array}$ & $\begin{array}{l}\text { Di- } \\
\text { eldrin }\end{array}$ & DDE & Endrin & $\begin{array}{l}\beta- \\
\text { Endo }\end{array}$ & DDD & $\begin{array}{l}\text { Endrin- } \\
\text { aldehyde }\end{array}$ & $\begin{array}{l}\text { Endo- } \\
\text { Sulfate }\end{array}$ & DDT & $\begin{array}{l}\text { Methoxy } \\
\text { Chlor }\end{array}$ \\
\hline TW-1 & ND & 0.004 & ND & ND & ND & 0.012 & ND & 0.024 & ND & 0.017 & ND & ND & ND & ND & ND & 0.008 & ND \\
\hline TW-2 & 0.017 & 0.045 & 0.057 & 0.018 & 0.018 & 0.047 & ND & ND & ND & ND & ND & ND & ND & ND & ND & 0.035 & ND \\
\hline TW-3 & 0.011 & 0.024 & 0.036 & 0.007 & 0.007 & 0.029 & ND & ND & ND & ND & ND & ND & ND & ND & ND & 0.015 & ND \\
\hline TW-4 & ND & ND & 0.012 & ND & ND & 0.010 & ND & ND & ND & ND & ND & 0.013 & ND & ND & ND & ND & ND \\
\hline TW-5 & ND & ND & ND & ND & ND & 0.022 & ND & ND & ND & ND & ND & 0.009 & ND & ND & ND & ND & 0.041 \\
\hline TW-6 & ND & ND & ND & ND & ND & ND & ND & ND & ND & ND & ND & ND & ND & ND & ND & ND & ND \\
\hline TW-7 & 0.014 & 0.079 & 0.055 & 0.078 & 0.022 & 0.009 & 0.019 & 0.020 & 0.066 & 0.066 & 0.041 & 0.024 & 0.064 & 0.052 & 0.074 & 0.082 & 0.089 \\
\hline TW-8 & ND & ND & ND & ND & ND & ND & ND & ND & ND & ND & 0.058 & ND & 0.030 & ND & ND & ND & ND \\
\hline TW-9 & ND & ND & ND & ND & ND & ND & ND & ND & ND & ND & ND & ND & ND & ND & ND & ND & ND \\
\hline TW-10 & 0.012 & 0.018 & 0.028 & 0.012 & ND & 0.033 & ND & ND & 0.009 & 0.009 & ND & ND & ND & 0.044 & ND & ND & ND \\
\hline TW-11 & 0.015 & 0.089 & 0.055 & 0.078 & 0.017 & 0.009 & 0.021 & 0.020 & 0.075 & 0.033 & 0.048 & 0.045 & 0.033 & 0.047 & 0.044 & 0.052 & 0.066 \\
\hline TW-12 & 0.005 & 0.014 & 0.013 & ND & ND & 0.015 & ND & ND & ND & 0.009 & ND & ND & 0.011 & ND & ND & ND & ND \\
\hline TW-13 & 0.004 & 0.008 & 0.013 & 0.005 & 0.022 & 0.016 & 0.012 & ND & ND & 0.014 & ND & 0.030 & 0.007 & ND & ND & 0.018 & 0.050 \\
\hline TW-14 & 0.015 & 0.201 & 0.041 & 0.012 & 0.017 & 0.015 & 0.009 & 0.015 & ND & 0.010 & ND & 0.020 & 0.013 & 0.059 & ND & ND & 0.034 \\
\hline TW-15 & 0.018 & 0.033 & 0.061 & 0.022 & 0.019 & 0.042 & ND & ND & ND & 0.010 & 0.018 & 0.050 & 0.012 & 0.069 & ND & ND & ND \\
\hline TW-16 & ND & 0.009 & ND & ND & ND & ND & ND & ND & ND & 0.024 & ND & ND & ND & ND & ND & 0.014 & ND \\
\hline TW-17 & ND & ND & ND & ND & ND & $\mathrm{ND}$ & ND & 0.012 & ND & ND & ND & 0.017 & ND & ND & ND & ND & ND \\
\hline TW-18 & 0.007 & 0.017 & ND & 0.012 & 0.022 & 0.013 & 0.012 & ND & ND & 0.008 & ND & 0.029 & ND & ND & ND & ND & 0.075 \\
\hline TW-19 & 0.008 & 0.016 & 0.025 & 0.010 & 0.016 & 0.016 & 0.008 & ND & ND & 0.011 & ND & ND & 0.014 & ND & ND & 0.037 & 0.034 \\
\hline TW-20 & ND & ND & ND & ND & ND & 0.014 & ND & ND & 0.015 & ND & 0.021 & 0.016 & ND & ND & ND & ND & ND \\
\hline TW-21 & 0.012 & 0.034 & 0.048 & 0.015 & 0.018 & 0.030 & 0.009 & 0.019 & 0.018 & 0.013 & 0.021 & 0.094 & 0.016 & 0.053 & 0.020 & 0.035 & 0.035 \\
\hline
\end{tabular}

Table 6. Organochlorine residues $\left(\mu \mathrm{g} 1^{-1}\right)$ in ground water (Ranny well) of Palla area during pre-monsoon seasons.

\begin{tabular}{|c|c|c|c|c|c|c|c|c|c|c|c|c|c|c|c|c|c|}
\hline & $\begin{array}{l}\alpha- \\
\mathrm{HCH}\end{array}$ & $\begin{array}{l}\beta- \\
\mathrm{HCH}\end{array}$ & $\begin{array}{l}\gamma- \\
\mathrm{HCH}\end{array}$ & $\begin{array}{l}\delta- \\
\mathrm{HCH}\end{array}$ & $\begin{array}{l}\text { Hepta } \\
\text { chlor }\end{array}$ & Aldrin & $\begin{array}{l}\text { Hepta- } \\
\text { Epoxide }\end{array}$ & $\begin{array}{l}\alpha- \\
\text { Endo }\end{array}$ & $\begin{array}{l}\text { Di- } \\
\text { eldrin }\end{array}$ & DDE & Endrin & $\begin{array}{l}\beta- \\
\text { Endo }\end{array}$ & DDD & $\begin{array}{l}\text { Endrin- } \\
\text { aldehyde }\end{array}$ & $\begin{array}{l}\text { Endo- } \\
\text { Sulfate }\end{array}$ & DDT & $\begin{array}{l}\text { Methoxy } \\
\text { Chlor }\end{array}$ \\
\hline RW-1 & 0.003 & 0.012 & 0.016 & 0.008 & ND & 0.013 & ND & ND & ND & 0.009 & ND & 0.015 & ND & ND & ND & ND & ND \\
\hline RW-2 & 0.006 & 0.017 & 0.027 & 0.011 & ND & 0.030 & ND & ND & 0.010 & 0.009 & ND & 0.020 & ND & 0.035 & ND & ND & 0.033 \\
\hline RW-3 & 0.012 & 0.022 & 0.035 & 0.015 & 0.015 & 0.021 & 0.008 & ND & ND & 0.009 & ND & 0.022 & 0.012 & ND & ND & ND & 0.034 \\
\hline RW-4 & 0.014 & 0.206 & 0.032 & 0.012 & 0.017 & 0.015 & 0.009 & 0.015 & ND & 0.011 & ND & ND & 0.014 & ND & ND & 0.037 & 0.035 \\
\hline RW-5 & 0.016 & 0.099 & 0.069 & 0.100 & 0.027 & 0.030 & 0.041 & 0.067 & 0.115 & 0.073 & 0.083 & 0.095 & 0.073 & 0.087 & 0.066 & 0.072 & 0.070 \\
\hline
\end{tabular}

were below their regulatory concentrations in the borewells. $\alpha$-endosulfan was present only in one sample $\left(0.034 \mu \mathrm{g} \mathrm{l}^{-1}\right)$, which shows the wise use of highly controversial pesticide, endosulfan in Yamuna catchment area. In case of Ranney well samples, the trends of occurrences were similar to the pre-monsoon sampling campaign; but the levels were lower (Table 8, Fig. 5). The student's t-test was carried out for pre-monsoon and post-monsoon samples taking all the samples as independent variables. It showed that there was no significant difference in total pesticide concentration in both the season $(P>0.05)$. Thus, it can be inferred that during whole year people receiving water supply from this wellfield are at high risk of pesticide exposure. Though, levels in post-monsoon season were lower than safe limit, but were not statistically different from pre-monsoon season. However, concentration of individual pesticides for both the seasons was statistically different $(P<0.01)$ for borewells and Ranney wells. This indicates that concentrations of individual pesticides decreased in post-monsoon season. It can be attributed to the dilution due to recharge of the aquifer during monsoon. Figures 6 and 7 indicate the total pesticides concentration in individual samples. The water quality of borewells was superior to the Ranney well as none of the borewell sample was found to contain the OCP residues level more than BIS permissible limit (Figs. 6 and 7). As per BIS drinking water standard, the water should be free from all pesticides, but in case of non availability of other sources, the limit is $1 \mu \mathrm{g}{ }^{-1}$ of total pesticides. Water, other than packaged mineral water, is not an important commodity in international trade, so there is no initiative in place to harmonize these limits at the international level. It varies from place to place, depending on the awareness level and economy of the country. The safe limit for pesticide residues are different in different parts of the world: WHO and EU have a safe limit of $0.5 \mu \mathrm{g}{ }^{-1}$ as compare to BIS limit of $1 \mu \mathrm{gl}^{-1}$. Recent reports on the total OCP levels in groundwater of neighbouring areas of Palla (Delhi) have reported much higher concentrations, $2.184 \mu \mathrm{g}^{-1}$ in Haryana (Kaushik et al., 2011) and up to $4 \mu \mathrm{g}^{-1}$ in Lucknow city (Mudiam et al., 2011). This wellfield is being recharged with the bank filtrate of river Yamuna (Lorenzen et al., 2010). Both external flux through bank filtrate and local use of pesticides can contribute to the pesti- 
Table 7. Organochlorine residues $\left(\mu \mathrm{g}^{-1}\right)$ in ground water (bore well) of Palla area during post-monsoon season.

\begin{tabular}{|c|c|c|c|c|c|c|c|c|c|c|c|c|c|c|c|c|c|}
\hline & $\begin{array}{l}\alpha- \\
\mathrm{HCH}\end{array}$ & $\begin{array}{l}\beta- \\
\mathrm{HCH}\end{array}$ & $\begin{array}{l}\gamma- \\
\mathrm{HCH}\end{array}$ & $\begin{array}{l}\delta- \\
\mathrm{HCH}\end{array}$ & $\begin{array}{l}\text { Hepta } \\
\text { chlor }\end{array}$ & Aldrin & $\begin{array}{l}\text { Hepta- } \\
\text { Epoxide }\end{array}$ & $\begin{array}{l}\alpha- \\
\text { Endo }\end{array}$ & $\begin{array}{l}\text { Di- } \\
\text { eldrin }\end{array}$ & DDE & Endrin & $\begin{array}{l}\beta \text { - } \\
\text { Endo }\end{array}$ & DDD & $\begin{array}{l}\text { Endrin- } \\
\text { aldehyde }\end{array}$ & $\begin{array}{l}\text { Endo- } \\
\text { Sulfate }\end{array}$ & DDT & $\begin{array}{l}\text { Methoxy } \\
\text { Chlor }\end{array}$ \\
\hline TW-1 & 0.004 & 0.009 & ND & ND & ND & 0.024 & ND & ND & ND & ND & ND & ND & ND & ND & ND & ND & ND \\
\hline TW-2 & ND & ND & ND & ND & ND & ND & ND & ND & ND & ND & ND & ND & ND & 0.040 & ND & ND & ND \\
\hline TW-4 & ND & ND & 0.007 & ND & ND & ND & ND & ND & ND & ND & ND & ND & ND & ND & ND & ND & ND \\
\hline TW-5 & ND & ND & ND & ND & ND & 0.019 & ND & ND & ND & ND & ND & ND & ND & ND & ND & ND & ND \\
\hline TW-6 & ND & ND & ND & ND & ND & ND & ND & ND & ND & ND & ND & ND & ND & ND & ND & ND & ND \\
\hline TW-7 & 0.005 & 0.037 & 0.012 & 0.039 & 0.032 & 0.034 & 0.026 & 0.034 & 0.058 & 0.046 & 0.046 & 0.041 & 0.055 & 0.069 & 0.057 & 0.093 & 0.094 \\
\hline TW-10 & 0.003 & ND & ND & ND & ND & 0.011 & ND & ND & ND & ND & ND & ND & ND & ND & ND & ND & ND \\
\hline TW-11 & ND & ND & ND & ND & ND & 0.014 & ND & ND & ND & ND & ND & ND & ND & ND & ND & ND & ND \\
\hline TW-12 & ND & ND & ND & ND & ND & ND & ND & ND & 0.009 & 0.018 & ND & ND & ND & ND & ND & ND & ND \\
\hline TW-13 & ND & ND & ND & ND & ND & 0.018 & ND & ND & ND & 0.018 & ND & ND & 0.022 & ND & ND & ND & 0.066 \\
\hline TW-14 & ND & ND & ND & ND & ND & ND & ND & ND & ND & ND & 0.029 & ND & ND & 0.083 & ND & ND & ND \\
\hline TW-15 & ND & 0.013 & 0.009 & 0.014 & 0.028 & 0.025 & 0.017 & ND & 0.016 & 0.021 & ND & ND & 0.025 & ND & 0.038 & 0.069 & 0.069 \\
\hline TW-16 & ND & 0.008 & ND & ND & ND & ND & ND & ND & ND & ND & ND & ND & ND & ND & ND & ND & ND \\
\hline
\end{tabular}

Table 8. Organochlorine residues $\left(\mu \mathrm{gl}^{-1}\right)$ in ground water (Ranny well) of Palla area during post-monsoon seasons.

\begin{tabular}{|c|c|c|c|c|c|c|c|c|c|c|c|c|c|c|c|c|c|}
\hline & $\begin{array}{l}\alpha- \\
\mathrm{HCH}\end{array}$ & $\begin{array}{l}\beta- \\
\mathrm{HCH}\end{array}$ & $\begin{array}{l}\gamma- \\
\mathrm{HCH}\end{array}$ & $\begin{array}{l}\delta- \\
\mathrm{HCH}\end{array}$ & $\begin{array}{l}\text { Hepta } \\
\text { chlor }\end{array}$ & Aldrin & $\begin{array}{l}\text { Hepta- } \\
\text { Epoxide }\end{array}$ & $\begin{array}{l}\alpha- \\
\text { Endo }\end{array}$ & $\begin{array}{l}\text { Di- } \\
\text { eldrin }\end{array}$ & DDE & Endrin & $\begin{array}{l}\beta- \\
\text { Endo }\end{array}$ & DDD & $\begin{array}{l}\text { Endrin- } \\
\text { aldehyde }\end{array}$ & $\begin{array}{l}\text { Endo- } \\
\text { Sulfate }\end{array}$ & DDT & $\begin{array}{l}\text { Methoxy } \\
\text { Chlor }\end{array}$ \\
\hline RW-1 & 0.006 & ND & ND & 0.013 & ND & 0.018 & 0.016 & ND & 0.010 & 0.019 & ND & 0.018 & 0.024 & ND & 0.036 & 0.068 & 0.068 \\
\hline RW-2 & 0.005 & 0.011 & 0.037 & 0.022 & ND & 0.018 & ND & ND & ND & ND & ND & 0.023 & ND & 0.015 & ND & ND & ND \\
\hline RW-3 & 0.005 & ND & 0.024 & ND & ND & 0.021 & ND & 0.024 & ND & ND & ND & 0.022 & 0.022 & ND & ND & ND & ND \\
\hline RW-4 & 0.009 & 0.015 & 0.019 & ND & ND & ND & ND & ND & ND & 0.013 & ND & ND & ND & ND & ND & ND & 0.068 \\
\hline RW-5 & 0.008 & 0.015 & 0.018 & 0.017 & ND & 0.018 & 0.019 & 0.044 & 0.021 & 0.023 & 0.030 & 0.032 & 0.031 & 0.018 & 0.038 & 0.074 & 0.070 \\
\hline
\end{tabular}

cides in the aquifer. The presence of $\alpha-\mathrm{HCH}$ and $\gamma-\mathrm{HCH}$ reveals the use of technical $\mathrm{HCH}(55-80 \% \alpha-\mathrm{HCH}$ and $8-$ $15 \% \gamma$-HCH plus other compounds) and lindane (more than $99 \% \gamma-\mathrm{HCH}$ ) in the Yamuna catchment area. Most of the developed countries have banned the use of technical $\mathrm{HCH}$ in the 1980s, but in India, it is still under production for export and restricted use, other than agriculture. The $\alpha-\mathrm{HCH} / \gamma$ $\mathrm{HCH}$ ratio within the technical formulation ranges from 4-15 and $0.2-1$ in lindane (Ridal et al., 1996). The low ratios of $\alpha-\mathrm{HCH} / \gamma-\mathrm{HCH}$ (Table 9) in the ground water samples indicate that lindane may also be an important source of HCHs in Yamuna river watershed. Among the DDTs, 4, 4-DDD and DDE were found to be the predominant compound (Tables 5, 6, 7 and 8). The DDE/DDTs and DDD/DDTs ratios can be used to assess whether the DDT input is of recent origin or from the past (Kannan et al., 1997). Since DDE/DDTs and DDD/DDTs ratios in all the samples were much lower than unity (Table 9), it appears DDT inputs is of recent origin from the Yamuna river catchment area. Total annual production of DDT in India is still more than $3000 \mathrm{t}$. (UNEP/POPS, 2008). It is used for export and malaria control programme in India, which supports the findings of recent inputs of DDT.
Table 9. Concentration ratios of selected OCP's compounds in the ground water according to the sampling source and seasons.

\begin{tabular}{llllll}
\hline \multicolumn{2}{c}{ Sampling sites } & $\begin{array}{l}\alpha \text {-HCH/ } \\
\gamma \text {-HCH }\end{array}$ & $\begin{array}{l}\text { DDE/ } \\
\text { DDTs }\end{array}$ & $\begin{array}{l}\text { DDD/ } \\
\text { DDTs }\end{array}$ & $\begin{array}{l}\text { DDE/ } \\
\text { DDD }\end{array}$ \\
\hline Borewell & Pre-monsoon & 0.245 & 0.518 & 0.269 & 0.931 \\
& Post-monsoon & 0.347 & 0.692 & 0.327 & 1.334 \\
\hline \multirow{2}{*}{ Ranney well } & Pre-monsoon & 0.292 & 0.319 & 0.319 & 1.137 \\
& Post-monsoon & 0.329 & 0.274 & 0.2 & 0.709 \\
\hline
\end{tabular}

The DDE/DDD ratio is indicative of the prevalent redox conditions in the area. While, in oxic conditions, DDE is the main metabolite of DDT (Wolfe et al., 1977), in anoxic conditions, DDD is the major degradation product (Zoro et al., 1974). Here, the DDE/DDD ratio varies from 0.7091.334 , more than unity is indicative of the less reducing conditions or an extended degradation during the long- range transport of DDT. As the major share of the ground water is replenished by river Yamuna and the river is in losing condition (Lorenzen et al., 2010) in this area, the background level of OCP present in river water could also reach into 


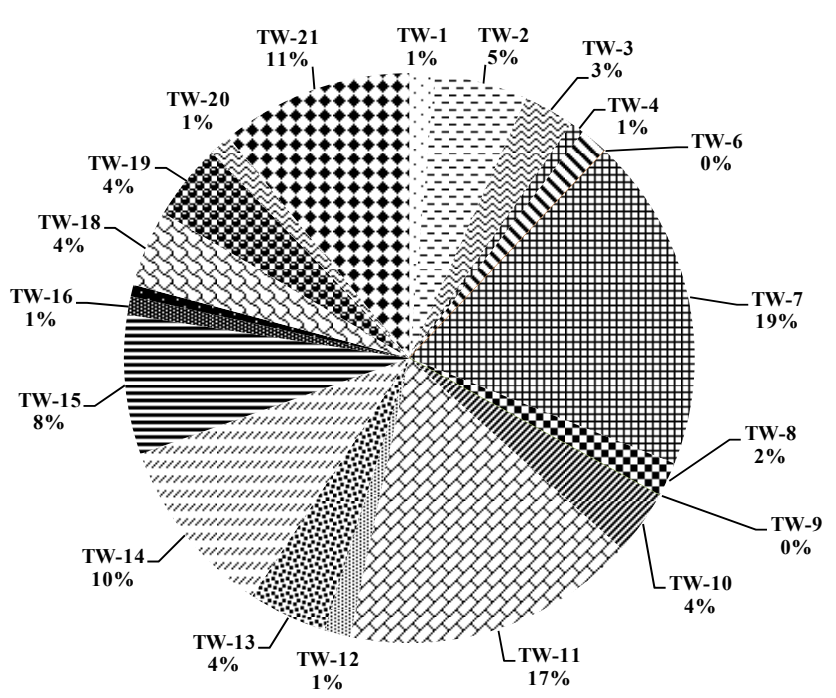

Figure 2. Relative abundance of OCP in different borewell samples (Pre-monsoon).

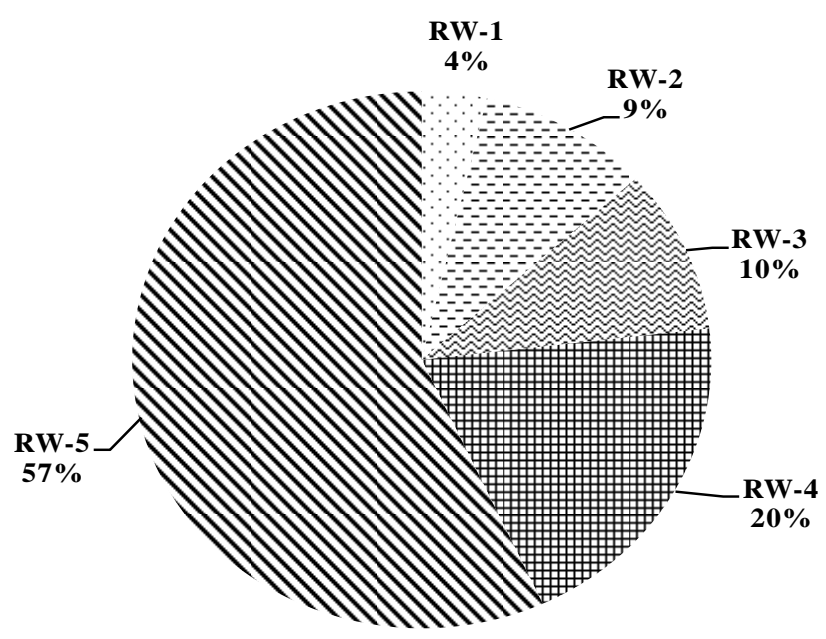

Figure 3. Relative abundance of OCP in different Ranney well samples (Pre-monsoon).

the aquifers. Kaushik et al. (2008), monitored the levels of OCP's in the river Yamuna and reported $\sum \mathrm{HCH} 0.012$ $0.593 \mu \mathrm{g}^{-1}$, and $\sum \mathrm{DDT}=0.066-0.723 \mu \mathrm{gl}^{-1}$ in surface water of river Yamuna, in upstream of this well-field. Levels of these pesticides in the aquifer of this well-field were much lesser. It may be because of dilution or adsorption of organic compounds by soil strata during recharge. The high affinity to soil organic matter (high $K_{\mathrm{OC}}$ ) make these pesticides strongly bound to soil organic matter and thus they are less leached in the aquifer while recharging the well-field. The levels of OCP residues were higher for Ranney wells than borewells (Tables 5, 6, 7 and 8) in all the cases. Ranney well have much higher hydraulic gradient as compared to borewells. Thus, higher gradient may result in higher desorp-

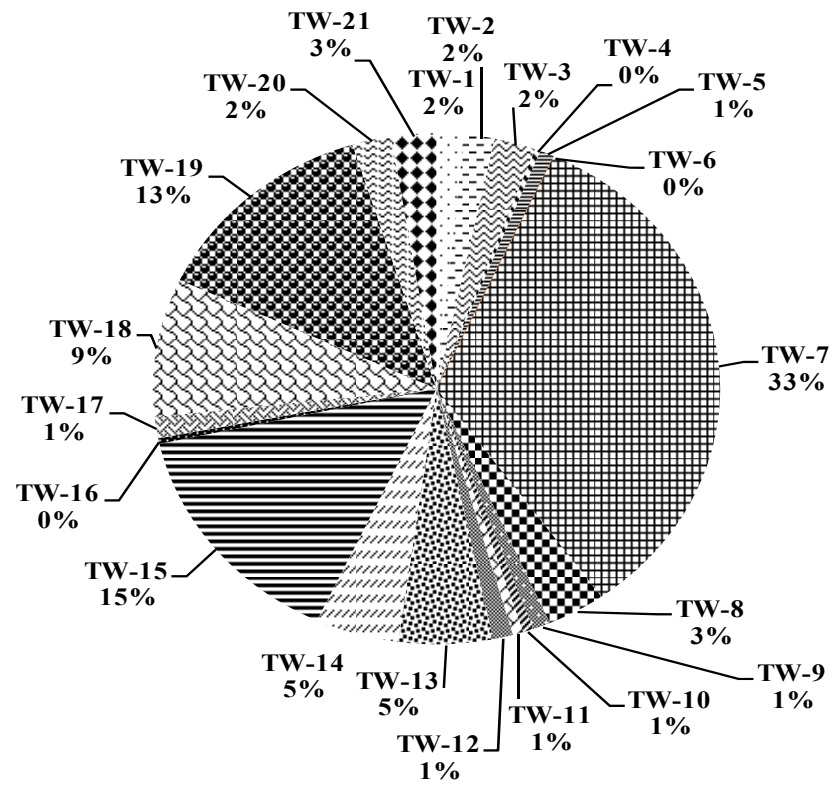

Figure 4. Relative abundance of OCP in different borewell samples (Post-monsoon).

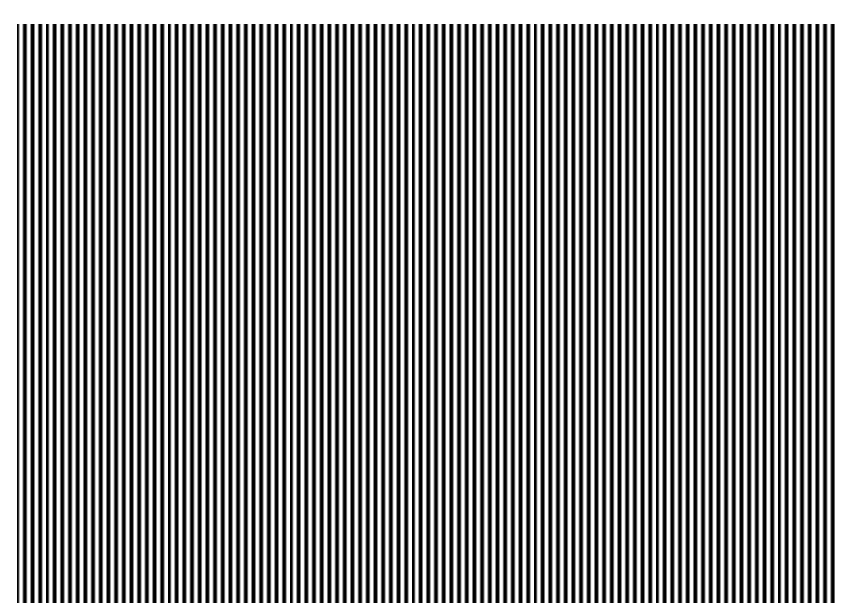

Figure 5. Relative abundance of OCP in different Ranney well samples (Post-monsoon).

tion of pesticides from solid phase to aquifer or lower transfer of pesticides from aquifer to soil particles.

\section{Conclusions}

There is wide prevalence of OCPs throughout the wellfield though at low concentrations. During monsoon high flood levels results in recharge of the well-field. But, there is no statistical difference between pre-monsoon and postmonsoon periods. The borewell water quality was superior to the Ranney well. So it is advantageous to develop a wellfield with borewells rather using Ranney wells. Although the levels of pesticides were lower than the local regulatory 


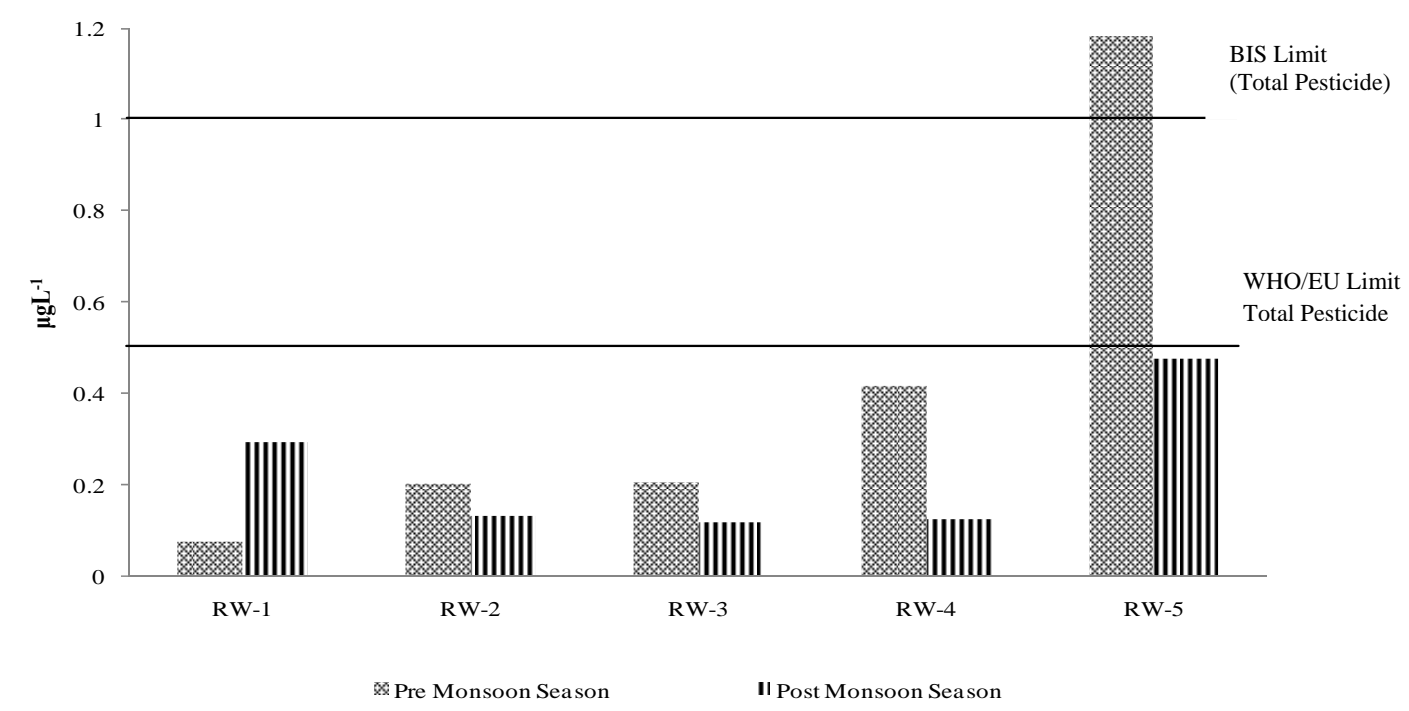

Figure 6. Comparison of Ranney well water quality with respect to various limits.

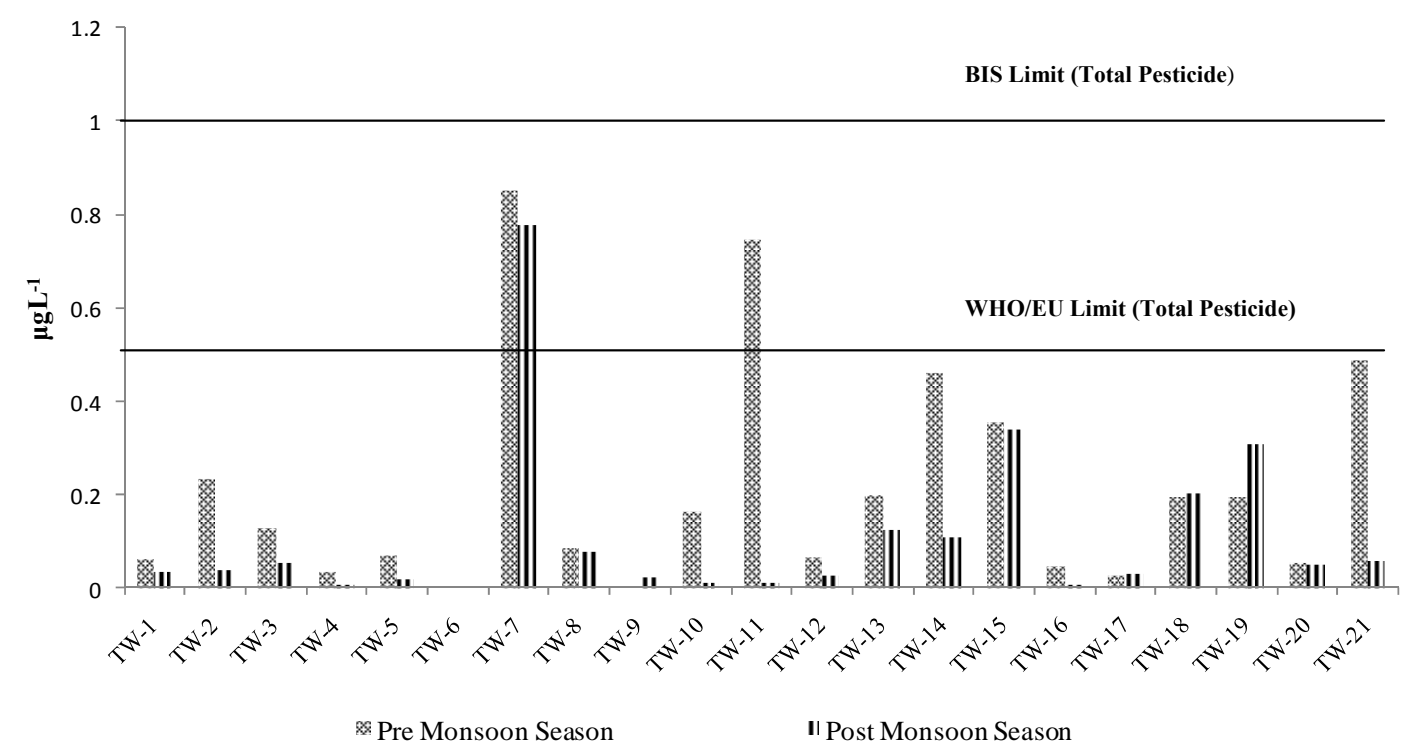

Figure 7. Comparison of borewell water quality with respect to various limits.

limits but regular consumption of such contaminated water may pose serious health hazard to the people who depend for the water on this well-field. In order to rule out any possibility of health risk by OCPs, regular monitoring of pesticide residues in river water and ground water is required. Delhi is a rapid growing urban centre, so there is a great political and social pressure to utilize this well-field to supply water for the city of Delhi since this well-field produces 6076 million $\mathrm{m}^{3}$ day $^{-1}$ potable water in all the seasons. There is need for comprehensive investigations to monitor the breakdown products of the pesticides in order to get better idea of the overall risk due to well contamination (since breakdown products are often toxic), and the mechanism causing wells to be contaminated.
Acknowledgements. One of the authors (Pravin K. Mutiyar) is thankful to University Grants Commission (UGC), New Delhi (India) for providing financial assistance in the form of Junior Research Fellowship (JRF) to conduct this work.

Edited by: J. Cho

\section{References}

American Public Health Association (APHA): Standard Methods for the Examination of Water and Wastewater, 20th edn., USA, 1998.

Bajpai, A., Shukla, P., Dixit, B., and Banerji, R.: Concentrations of organochlorine insecticides in edible oils from different regions 
of India, Chemosphere, 67, 1403-1407, 2007.

Bakore, N., John, P., and Bhatnagar, P.: Organochlorine pesticide residues in wheat and drinking water samples from Jaipur, Rajasthan, India, Environ. Monit. Assess., 98, 381-389, 2004.

Barrie, L. A., Gregor, D., Hargrave, B., Lake, R., Muir, D., Shearer, R., Tracey, B., and Bidleman, T.: Arctic contaminants: Sources, occurrence and pathways, Sci. Total Environ., 122, 1-74, 1992.

Bizzotto, E., Villa, S., Vaj, C., and Vighi, M.: Comparison of glacial and non-glacial-fed streams to evaluate the loading of persistent organic pollutants through seasonal snow/ice melt, Chemosphere, 74, 924-930, 2009.

Blais, J. M., Macdonald, R. W., Mackay, D., Webster, E., Harvey, C., and Smol, J. P.: Biologically mediated transport of contaminants to aquatic systems, Environ. Sci. Technol., 41, 1075-1084, 2007.

Dikshith, T., Raizada, R., Kumar, S., Srivastava, M., Kulshrestha, S., and Adholia, U.: Residues of DDT and $\mathrm{HCH}$ in major sources of drinking water in Bhopal, India, Bull. Environ. Contam. Toxicol., 45, 389-393, 1990.

Dua, V., Pant, C., and Sharma, V.: HCH and DDT in surface extractable skin lipid as a measure of human exposure in India, Bull. Environ. Contam. Toxicol., 60, 238-244, 1998.

Geisz, H. N., Dickhut, R. M., Cochran, M. A., Fraser, W. R., and Ducklow, H. W.: Melting glaciers: A probable source of DDT to the Antarctic marine ecosystem, Environ. Sci. Technol., 42, 3958-3962, 2008.

Hassan, A. A. M., Benfenati, E., and Fanelli, R.: Detection and quanification of trihalomethanes in drinking water from Alexandria, Egypt, Bull. Environ. Contam. Toxicol., 56, 397-404, 1996.

Jani, J., Raiyani, C., Mistry, J., Patel, J., Desai, N., and Kashyap, S.: Residues of organochlorine pesticides and polycyclic aromatic hydrocarbons in drinking water of Ahmedabad city, India, Bull. Environ. Contam. Toxicol., 47, 381-385, 1991.

John, P., Bakore, N., and Bhatnagar, P.: Assessment of organochlorine pesticide residue levels in dairy milk and buffalo milk from Jaipur city, Rajasthan, India, Environ. Int., 26, 231-236, 2001.

Kannan, K., Tanabe, S., Giesy, J. P., and Tatsukawa, R.: Organochlorine pesticides and polychlorinated biphenyls in foodstuffs from asian and oceanic countries, Rev. Environ. Contam. Toxicol., 152, 1-56, 1997.

Kaushik, C., Sharma, H., Jain, S., Dawra, J., and Kaushik, A.: Pesticide residues in river Yamuna and its canals in Haryana and Delhi, India, Environ. Monit. Assess., 144, 329-340, 2008.

Kaushik, C., Sharma, H., and Kaushik, A.: Organochlorine pesticide residues in drinking water in the rural areas of Haryana, India, Environ. Monit. Assess., doi:10.1007/s10661-011-1950-9, in press, 2011.

Kumar, A., Dayal, P., Shukla, G., Singh, G., and Joseph, P.: DDT and $\mathrm{HCH}$ residue load in mother's breast milk: A survey of lactating mother's from remote villages in Agra region, Environ. Int., 32, 248-251, 2006a.

Kumar, M., Ramanathan, A., Rao, M., and Kumar, B.: Identification and evaluation of hydrogeochemical processes in the groundwater environment of Delhi, India, Environ. Geol., 50, 1025-1039, 2006b.
Lorenzen, G., Sprenger, C., Taute, T., Pekdeger, A., Mittal, A., and Massmann, G.: Assessment of the potential for bank filtration in a water-stressed megacity (Delhi, India), Environ. Earth Sci., 61, 1419-1433, 2010.

Mudiam, M. K. R., Pathak, S., Gopal, K., and Murthy, R.: Studies on urban drinking water quality in a tropical zone, Environ. Monit. Assess., doi:10.1007/s10661-011-1980-3, in press, 2011.

Mukherjee, I. and Gopal, M.: Insecticide residues in baby food, animal feed, and vegetables by gas liquid chromatography, Bull Environ. Contam. Toxicol., 56, 381-388, 1996.

Mukherjee, I. and Gopal, M.: Organochlorine insecticide residues in drinking and ground water in and around Delhi, Environ. Monit. Assess., 76, 185-193, 2002.

Ridal, J. J., Kerman, B., Durham, L., and Fox, M. E.: Seasonality of air-water fluxes of hexachlorocyclohexanes in Lake Ontario, Environ. Sci. Technol., 30, 852-858, 1996.

Sankararamakrishnan, N., Kumar Sharma, A., and Sanghi, R.: Organochlorine and organophosphorous pesticide residues in ground water and surface waters of Kanpur, Uttar Pradesh, India, Environ. Int., 31, 113-120, 2005.

Sharma, H., Kaushik, A., and Kaushik, C.: Pesticide residues in bovine milk from a predominantly agricultural state of Haryana, India, Environ. Monit. Assess., 129, 349-357, 2007.

Shekhar, S. and Prasad, R.: The groundwater in the Yamuna flood plain of Delhi (India) and the management options, Hydrogeol. J., 17, 1557-1560, 2009.

Shukla, G., Kumar, A., Bhanti, M., Joseph, P., and Taneja, A.: Organochlorine pesticide contamination of ground water in the city of Hyderabad, Environ. Int., 32, 244-247, 2006.

UNEP/POPS: Global status of DDT and its alternatives for use in vector control to prevent disease, 2008.

Villa, S., Vighi, M., Maggi, V., Finizio, A., and Bolzacchini, E.: Historical trends of organochlorine pesticides in an Alpine glacier, J. Atmos. Chem., 46, 295-311, 2003.

Waliszewski, S., Aguirre, A., Infanzon, R., Silva, C., and Siliceo, J.: Organochlorine pesticide levels in maternal adipose tissue, maternal blood serum, umbilical blood serum, and milk from inhabitants of Veracruz, Mexico, Arch. Environ. Contam. Toxicol., 40, 432-438, 2001.

Wang, X., Xu, B., Kang, S., Cong, Z., and Yao, T.: The historical residue trends of DDT, hexachlorocyclohexanes and polycyclic aromatic hydrocarbons in an ice core from Mt. Everest, central Himalayas, China, Atmos. Environ., 42, 6699-6709, 2008.

Wolfe, N. L., Zepp, R. G., Paris, D. F., Baughman, G. L., and Hollis, R. C.: Methoxychlor and DDT degradation in water: Rates and products, Environ. Sci. Technol., 11, 1077-1081, 1977.

Zoro, J. A., Hunter, J. M., Eglinton, G., and Ware, G. C.: Degradation of p, p[prime]-DDT in reducing environments, Nature, 247, 235-237, 1974. 